ÉGYPTE

monde arabe

\section{Égypte/Monde arabe}

$34 \mid 1998$

Droits d'Égypte : histoire et sociologie

\title{
Entretien avec le conseiller Târiq al-Bishrî
}

Entretien conduit par 'Âmir 'Abd al-Mun'im pour al-Sha'b (21 et

28 mars 1997)

Traducteur : Muriel Paradelle

\section{OpenEdition}

\section{Journals}

Édition électronique

URL : https://journals.openedition.org/ema/1531

DOI : 10.4000/ema.1531

ISSN : 2090-7273

Éditeur

CEDEJ - Centre d'études et de documentation économiques juridiques et sociales

Édition imprimée

Date de publication : 31 décembre 1998

Pagination : 215-235

ISSN : 1110-5097

Référence électronique

"Entretien avec le conseiller Târiq al-Bishrî », Égypte/Monde arabe [En ligne], 34 | 1998, mis en ligne le 08 juillet 2008, consulté le 07 juillet 2022. URL : http://journals.openedition.org/ema/1531 ; DOI :

https://doi.org/10.4000/ema.1531

Ce document a été généré automatiquement le 7 juillet 2022.

Tous droits réservés 


\section{Entretien avec le conseiller Târiq al- Bishrî}

Entretien conduit par 'Âmir 'Abd al-Mun'im pour al-Sha'b (21 et

28 mars 1997)

Traduction : Muriel Paradelle

\section{Première partie :}

\section{" Les textes du Coran et de la Sunna n'ont jamais fait obstacle à l'exercice du raisonnement humain, ils posent seulement des principes encadrant son exercice » (21 mars 1997)}

1 De nombreux changements sont apparus sur le terrain de l'islam, qui connaît désormais des situations inédites, rendant l'usage du raisonnement absolument nécessaire afin de permettre l'harmonisation des prescriptions islamiques avec l'époque actuelle.

2 Le journal al-Sha'b s'est entretenu avec le conseiller Târiq al-Bishrî sur ce sujet. Celui-ci est considéré comme l'un des plus importants penseurs musulmans contemporains ${ }^{\circ}$; il occupe les fonctions de vice-président du Conseil d'État et de président de la Commission des avis législatifs de l'assemblée générale [de ce même Conseil]. Dans le cadre de ses fonctions, il a contribué de façon significative à l'approfondissement des questions juridiques, et, en sus de son travail au sein de la magistrature, il s'est également préoccupé d'histoire, écrivant avec compétence sur l'histoire de l'Egypte en faisant preuve à l'occasion de beaucoup d'honnêteté, sans jamais chercher à déformer la réalité, comme l'ont fait certains qui prétendaient retracer l'histoire.

3 Les contributions du conseiller Târiq al-Bishrî dans le domaine de la pensée islamique se sont révélées d'une extrême importance, dans la mesure où il s'est attaché à aborder des questions sur lesquelles Yijtihâd (effort de raisonnement) s'est lui-même attardé. Le journal al-Sha'b a entrepris un voyage intellectuel avec cet homme, afin de connaître son opinion sur certaines questions vivement débattues récemment - en raison du regain d'intensité que connaît aujourd'hui le conflit opposant les laïcs aux islamistes. 
Parmi ces questions, celle relative à la liberté de pensée et de raisonnement, et notamment le fait de savoir s'il peut être mis un frein ou non à cette dernière ${ }^{\circ}$; la possibilité d'un conflit entre les textes islamiques et les intérêts de la société est-elle envisageable ${ }^{\circ}$ ? Quel est le système juridictionnel de l'islam? Quelles sont les conditions d'application des hudûd (peines limitativement reconnues par la shari'a) ${ }^{\circ}$. Existe-t-il ou non une contradiction entre la démocratie et la shûrâ (système consultatif)? Quels sont les droits des coptes dans l'islam... ${ }^{\circ}$ ?

Al-Sha'b: Quelle est votre opinion concernant l'intensification de la controverse portant sur la liberté de pensée, laquelle fait partie des droits de l'homme, et que représente pour vous le fait de recourir au raisonnement, y compris lorsqu'un tel usage conduit à un conflit avec les textes ${ }^{\circ}$ ? Pensez-vous qu'il existe des restrictions à cette libertéo?

Târiq al-Bishrî : Est-il besoin une fois encore de rapporter ce propos, selon lequel aussi bien la religion que le Noble Coran ont toujours appelé et appellent toujours l'homme à user de sa raison. C'est en suivant cette ligne de conduite tracée par le Coran que nous parvenons à réaliser l'existence de Dieu, qu'il soit louéo: c'est en ayant recours à la raison que nous prenons conscience de l'existence de Dieu et du message prophétique et que nous pouvons alors nous soumettre aux principes de l'islam.

En premier lieu, le Coran contient nombre de versets qui incitent l'homme à la compréhension, à la rationalisation et à la prise de conscience à partir de ce qu'il perçoit des signes cosmiques révélateurs de la présence de Dieu dans la société des hommes. En second lieu, il me semble que poser la question de cette façon, à savoir s'il est possible de faire usage de la raison alors même que celle-ci viendrait à contredire les textes, revient à la poser dans des termes inadéquats, parce que la probabilité d'une contradiction entre i'usage de la raison et les textes n'existe pas. La croyance dans le message divin est une posture intellectuelle et philosophique qui renvoie à la relation de l'homme avec l'univers ${ }^{\circ}$; or il n'existe aucune doctrine religieuse ou philosophique qui, à un moment ou à un autre, ne définisse un certain nombre de postulats relatifs à cette posture, postulats qu'elles renforcent par des emprunts à la philosophie ainsi qu'à d'autres disciplines et qui renvoient au système socio-politique, aux relations humaines, etc. L'histoire de la philosophie tout entière depuis ses origines s'est construite sur un postulat essentiel, à partir duquel se fonde l'usage de la raison. C'est pourquoi j'aimerais dès à présent poser la question suivante $^{\circ}$ : est-ce que les textes représentent un empêchement ${ }^{\circ}$ ? Est-ce que les postulats et principes religieux qui figurent dans le Coran et la Sunna se posent en obstacles à l'usage de la raison par l'homme ou non'? Je ne crois pas que cela ait jamais été le cas. Il n'est jamais arrivé que des prescriptions ou des principes tirés du Coran ou de la Sunna se soient opposés à l'exercice par l'homme de sa pleine capacité de raisonnement ${ }^{\circ}$; bien au contraire, celui-ci a toujours été encouragé à en faire usage. Le Noble Coran comme les hadith (dires) du Prophète, et avec eux toute la pensée islamique, ont permis l'élaboration de deux sciences reposant essentiellement sur l'exercice de la raison ${ }^{\circ}$ il s'agit de la science des fondements de la jurisprudence islamique (usûl al-fiqh) et de la science de la terminologie des Traditions prophétiques (mustalahât al-hadîth).

La science des usûl al-figh est encore appelée al-fakhral-râzî- dénomination choisie par sheikh Mustafa 'Abd al-Râziq, que la paix soit sur lui - ou « logique des musulmans », et c'est en rapport à cette science que l'on a appelé al-Shâfi'î « l'Aristote de l'islam », 
pour avoir introduit la science de la logique auprès des musulmans. Comment exercer le raisonnement, comment comprendre et analyser les différents concepts, comment construire la sémantique des expressions..., toutes ces questions ont été abordées dans la science des usûl al-figh, selon une méthode rationnelle réfléchie et solidement fondée. À partir de cette construction, les juristes et les jurisconsultes (fuqahâ', spécialistes du figh) ont œuvré à l'élaboration de la législation islamique, y compris la législation positive.

La seconde science est celle des mustalahât al-hadith, qui renvoie à la méthode permettant d'authentifier les hadith du Prophète. Elle pose un ensemble de règles destinées à contrôler l'authenticité historique de ces hadîth... Je ne pense pas que la raison humaine soit parvenue à vérifier l'authenticité de tous les faits historiques comme elle est parvenue à le faire s'agissant des nobles hadith du Prophète. Nous avons eu recours à la science des mustalahât al-hadîth afin de contrôler l'authenticité des différents récits rapportant les événements de l'histoire passée ${ }^{\circ}$; au terme de cette vérification, nous n'avons conservé qu'un cinquième ou un quart des hadith attribués au Prophète. Pour authentifier les hadith, il existait des méthodes très strictes.

Ces deux sciences sont, pour l'essentiel, des sciences de la raison, qui reposent sur une trame solide faite de liens rationnels et dont les sources sont principalement le Coran et la Sunna... Nous, les musulmans, constituons la umma (communauté des croyants), laquelle est née d'un livre, le Coran. Tout ce que la umma a construit, elle l'a fait au moyen d'efforts rationnels, à partir des sources du Coran et de la Sunna. Nous ne trouvons rien dans l'islam qui fasse obstacle au libre exercice de l'ijtihâd, qui est, à l'inverse, encouragé. D'après toutes les sources, la liberté de pratiquer l'ijtihâd, comme aussi la liberté de pensée, doit s'exercer dans le cadre des méthodes de raisonnement qui sont acceptées et suivies par un esprit civilisé, quelle que soit par ailleurs la culture considérée.

Al-Sha'b: Les postulats ${ }^{\circ}$ : Est-ce que les méthodes de raisonnement constituent des freins [au libre exercice de l'ijtihâd ainsi qu'à la liberté de pensée] ${ }^{\circ}$ ?

T. al-Bishrî: Il existe un certain nombre de postulats à partir desquels s'organise l'effort de réflexion... Nous les trouvons dans l'islam comme dans toutes les autres religions divines... Nous les trouvons également dans les doctrines qui s'écartent de la religion et qui, elles aussi, posent d'entrée certains postulats ${ }^{\circ}$ : ainsi la négation de Dieu le Très-Haut constitue pour elles un postulat, de même que l'affirmation selon laquelle l'origine de l'être est la matière et non l'âme. Aucune doctrine philosophique ne s'est élaborée sans donner naissance à des postulats, sur la base desquels elle a construit sa pensée et à partir desquels elle a approfondi sa réflexion. Dans un cas comme dans l'autre, il s'agit de méthodes posées pour atteindre la vérité en partant de postulats déterminés. Nos postulats, quant à nous, renvoient à l'existence de Dieu le Glorieux et le Très-Haut et à la croyance dans le message de Muhammad. La croyance selon laquelle le Coran est la Parole de Dieu descendue du monde invisible vers nous..., de même que l'acceptation sans discussion de tout ce qui provient de Dieu le Glorieux et le Très-Haut constituent des postulats fondamentaux. Certains philosophes et jurisconsultes ont précisément définis ceux-ci, lesquels affirment la croyance en Dieu, ses anges, ses Livres et le jour du Jugement dernier. Abu Hamîd alGhazâlî, pour sa part, a déclaré que « le premier postulat consiste à ne point attribuer de faux propos à l'Envoyé de Dieu, que la bénédiction et le salut de Dieu soient sur lui, 
et d'accepter comme tel ce que le Prophète a dit, que la bénédiction et le salut de Dieu soient sur lui ». Je pense, quant à moi, que l'existence de Dieu, de même que le Coran et la Sunna sont des postulats fondamentaux, sur la base et la croyance desquels on devient musulman.

Al-Sha'b : Certaines personnes prétendent qu'il peut arriver qu'il y ait conflit entre les intérêts [de la société] et les textes [de l'islam] et elles avancent, à l'appui de leurs dires, l'épisode de la non-application par Omar Ibn al-Khattab du châtiment contre le vol durant l'année de famine. Pensez-vous que l'hypothèse de la contradiction soit possible ${ }^{\circ}$ ?

T. al-Bishrî: Le point de vue des mujtahidûn (praticiens de l'ijtihâd) de la pensée islamique sur cette question peut se résumer en deux positions. La première considère les intérêts [de la société] et leur importance par rapport aux textes, dès lors qu'il existe une contradiction entre eux ${ }^{\circ}$ : ainsi peut-on citer à titre d'exemple ce que fit l'imam al-Tûfî lorsqu'il eut à se prononcer sur l'existence d'une contradiction entre le texte et l'intérêt et qu'il affirma qu'il fallait en pareil cas accorder le texte à l'intérêt, mais ne jamais abroger le texte, dans la mesure où la shari'a (loi islamique) tout entière n'est qu'intérêt [pour l'homme] et parce que la shari'a nous a été révélée en vue de préserver tous nos intérêts et de les renforcer.

L'imam al-Tûfî s'est ainsi exprimé alors qu'il devait interpréter quarante hadith nawawî (hadith authentiques) ${ }^{\circ}$ : lorsqu'il parvint au numéro trente-deux, aux termes duquel il est dit "je ne te causerai point de tort si tu ne me causes point de tort ", l'imam affirma que cette disposition emportait l'approbation de l'ensemble des intérêts et qu'elle exigeait de nous, en tant que musulmans, que nous ne portions point préjudice à autrui ni par nos actes ni par nos pensées. De la sorte, lorsque nous interprétons n'importe quelle disposition de la shari'a issue du Coran ou de la Sunna, nous devons l'interpréter dans les termes de ce texte, qui enjoint de ne causer aucun dommage à autrui si autrui ne nous en cause aucun... Ce faisant, ce ne sont pas tous les intérêts qui sont ici consacrés, mais les seuls intérêts légitimes appréciés à l'aune des dispositions de ce hadith : " Tu ne causeras point de tort à autrui si autrui ne te cause point de tort ». Il nous revient de mettre en application ce hadith pour toutes les questions qui requièrent de notre part que nous interprétions les principes de la shari'a. Ainsi parle l'imam al-Tûfî lorsqu'il interprète ce hadîth.

Un groupe de jurisconsultes et de réformistes, apparus au $\mathrm{XX}^{\mathrm{e}}$ siècle et issus de l'École de la justice de la shari'a, tels cheikh Ahmad Ibrâhîm et cheikh 'Abd al-Wahhâb Khalaf ont adopté cette manière de voir et l'ont toujours confirmée dans leurs ouvrages. Celle-ci leur a ouvert la voie de l'ijtihâd leur a permis d'harmoniser les textes avec les événements en constant changement tels qu'ils existaient à leur époque. Ils se sont également appuyés, pour ce faire, sur une déclaration de Ibn alQayyim al-Jawziyya, à savoir que « la shari'a n'est qu'intérêt ». Il semble que l'apogée de la tendance de cette école ait été atteinte avec l'excellente thèse de cheikh Muhammad Mustafa Shalabî traitant de l'interprétation des principes [de raisonnement]. Il a synthétisé très clairement la conception de l'école. De fait, il a suivi les principes de raisonnement tels que contenus dans le Coran et la Sunna, de même que ceux émanant des actes des compagnons du prophète et il est toujours parvenu à établir un lien entre textes et intérêts.

Une autre école a vu le jour avec l'imam al-Shâtibî, qui a composé un ouvrage sur «les agréments» (ijtihâd al-muwâfaqât), dans lequel il affirme que les différents principes s'articulent tous autour de fondements généraux communs. Al-Shâtibî est 
l'un de ces jurisconsultes spécialiste des usûlal-fiqh (des fondements du fiqh) qui manifeste une surprenante capacité à extraire les principes pour ensuite les ancrer [dans la réalité] en suivant une méthode scientifique sérieuse, celle de l'induction (alistiqrâ).

Lorsqu'il a parlé des intérêts dans le cadre des fins ultimes de la sharî'a, il s'est livré à un effort de raisonnement inductif à partir de ce que l'on considère comme étant des intérêts communs ou finalités ultimes communes de la sharîa islamique tirées des dispositions et autres principes détaillés provenant de la sharî́a et du Coran. Il a déduit de cet ensemble de prescriptions que les intérêts vont dans le sens de la préservation de la religion, de l'individu, de la famille, de la raison, de la richesse..., l'effort inductif se faisant toujours à partir des principes issus du Coran et de la Sunna.

Ce faisant, il a placé la religion au cœur de sa compréhension des intérêts et des finalités de la sharî́a. Poser la question de cette façon rend la conception selon laquelle les intérêts peuvent s'opposer à la religion infondée, dans la mesure où intérêts et finalités sont extraits des mêmes textes... Il a su lier très sûrement les textes et notre conception des intérêts, et s'il est essentiel en islam de préserver la religion et de protéger la communauté musulmane, alors il nous revient de prendre en compte ces considérations s'agissant des intérêts qui nous préoccupent.

Les deux conceptions envisagent l'intérêt en partant de l'affirmation selon laquelle celui-ci est contenu dans les textes de la sharî'a. Pour ma part, je ne pense pas qu'un jurisconsulte ou une école quelconque parmi les écoles de fiqh islamique aient pu négliger de prendre en compte la question des intérêts dans leurs commentaires, exégèses et autres interprétations doctrinales. En revanche, demeure pour toutes ces écoles, la question du choix des méthodes spécifiques devant servir à prendre en compte les innovations et à réglementer l'interprétation.

\section{Al-Sha'b : Comment doit être conduit, selon vous, l'effort de renouveau et d'interprétation?}

T. al-Bishrî : L'interprétation, à mon sens, représente une façon d'appliquer un texte stable à une réalité qui, elle, est évolutive, dans la mesure où le texte est stable et circonscrit là où les événements sont infinis dans leur variété, leur grand nombre et leur évolution. L'acte d'interpréter lié à un tel contexte repose sur le principe de la compréhension des faits et de la réalité telle qu'envisagée par le fiqh, qui leur applique ensuite les principes révélés. L'interprète se doit de ne jamais perdre de vue le texte et de demeurer vigilant quant aux façons de l'interpréter et aux limites de son interprétation, de même qu'il ne doit jamais perdre de vue les faits et ce qui se passe dans la réalité, afin de leur appliquer au mieux les principes issus de ces textes.

Dans chaque école islamique, nous trouvons exposés les moyens de prendre en compte le renouveau... Ainsi, par exemple l'école hanafite a-t-elle eu fréquemment recours au raisonnement par analogie (qiyâs) et a pu de ce fait étendre le champ d'application de ces principes en vue d'intégrer les évolutions de la réalité... L'école malikite, quant à elle, a recours à ce que l'on nomme al-masâlih al-mursala (intérêts dont la définition a été transmise par les jurisconsultes sur la base de l'intérêt général), ce que l'imam Shâtibî, lui-même malikite, a par la suite explicité dans son exposé des finalités de la législation islamique.

Ibn Hanbal s'est appuyé sur de nombreux hadith et, après eux, a beaucoup utilisé la méthode de l'istishâb (méthode de raisonnement qui privilégie la continuité d'une 
solution donnée). Lorsque qu'un texte n'existe pas, le principe est alors celui de la liberté d'interprétation. D'où l'importance des prescriptions relatives à la prise en compte du renouveau dans l'école hanafite, lesquelles sont dues au fait qu'elles reposent sur l'istishâb posée comme étant une source parmi d'autres de la législation. Or le principe de l'istishâb ouvre en grand aux jurisconsultes la voie de l'interprétation. Pour ce qui est des Zaydites, qui rejettent le raisonnement par analogie, ils ont eux aussi recours au principe de l'istishâb, c'est pourquoi nous trouvons chez eux aussi des solutions en accord avec la réalité contemporaine et les principes détaillés.

Al-Sha'b: Comment interprétez-vous la non-application par Omar Ibn al-Khattab du châtiment contre le vol lors de l'année de la famine ${ }^{\circ}$ ?

T. al-Bishrî: Omar Ibn al-Khattab n'a pas supprimé la sanction, mais il a pris la mesure des conditions réelles d'application de celle-ci, de la même façon qu'il n'a jamais rejeté le jugement, ni non plus affirmé que la réalité avait dépassé le principe. Il a, en revanche, réalisé que la sanction prévue en matière de vol ne pouvait et ne devait s'appliquer que si les éléments constitutifs du crime étaient réunis ${ }^{\circ}$; or, ces éléments ne pouvaient se trouver réunis dès lors qu'existait un état de nécessité, la famine étant une situation entrant dans la catégorie de l'état de nécessité. Le jugement de la sharî́a pose un exemple qu'il convient de répéter dans toutes ses conditions $^{\circ}$; lorsque je dis que le voleur doit être châtié, encore faut-il définir ce que l'on entend par "voleur». Dans le figh, nous trouvons précisés les éléments constitutifs de l'infraction, qui sont l'élément moral, l'élément matériel et l'élément légal. S'agissant de l'élément moral, il renvoie à l'intention'; l'élément matériel suppose l'acte constitutif du crime ${ }^{\circ}$; l'élément légal suppose quant à lui l'existence préalable d'une incrimination juridique. Or, si l'on se trouve dans un cas de nécessité, l'élément intentionnel de l'infraction fait défaut. Omar Ibn al-Khattab s'est borné, s'agissant de ce texte, à l'interpréter, non pour en contester le bien-fondé ou l'écarter, mais pour en rechercher toutes les circonstances et les conditions d'application. Voilà ce qu'a fait Omar.

Al-Sha'b: Certains se servent de la notion de masâlih mursala pour faire tomber sous le coup de la sharl'a des questions qui ne la concernent pas.

T. al-Bishrî: Les masâlih mursala sont les intérêts au sujet desquels le législateur n'a donné ni autorisation ni prohibition, aussi tombent-ils dans la catégorie des actes autorisés. Catégorie qui, elle, est régie par la sharîa. Par la méthode de l'induction, nous pouvons parvenir, en partant de l'esprit de la sharî'a et de ses fins ultimes, à déterminer les principes à même de contrôler et réguler l'application de ces intérêts. En effet, cette catégorie n'est pas une catégorie ouverte un peu comme une «zone libre ", elle est au contraire soumise aux restrictions posées par les principes légaux de l'interprétation, à savoir la préservation de la religion et la protection de la communauté.

Al-Sha'b: De nombreux changements ont eu lieu et tous les jours on peut relever des choses nouvelles. Selon vous, la pensée islamique a-t-elle besoin d'une reformulation ainsi que de nouvelles thèses, pour s'adapter à la réalité et pouvoir ainsi suivre les changements ${ }^{\circ}$ ?

T. al-Bishrî: La pensée islamique contemporaine, me semble-t-il, n'a jamais manqué de prendre en considération les changements survenus dans la réalitéo; trois vagues de renouveau se sont ainsi succédé dans la pensée islamique sur une période de deux 
siècles. La première vague de renouveau a commencé entre la fin du XVIIIe et le début du XIX siècle $^{\circ}$; elle prônait un retour aux principes fondamentaux, ceux du Coran et de la Sunna, et engageait à dépasser, ce faisant, le piège dans lequel étaient tombés, durant le Moyen Âge, les différentes écoles juridiques islamiques ainsi que les Soufis en reconnaissant la primauté de certaines personnes appelées «amis de Dieu ".

En effet, la naissance du mouvement du renouveau doctrinal et intellectuel s'explique au regard de la volonté d'aller au-delà des pratiques et des expériences confrériques soufies, notamment des idées qui avaient envahi la doctrine consacrée par ces écoles et conduit à leur sectarisme. Le renouveau s'est dès lors voulu un retour aux sources du Coran et de la Sunna en même temps qu'un engagement à la modération dans les expériences intellectuelles, afin d'éviter de tomber dans les excès. Relèvent de ce courant des penseurs comme Muhammad 'Abd al-Wahhâb, al-Imam al-Shawkânîu encore Shihâb al-Alûsî d'Irak et Walî al-Dîn al-Dahlâwî en Inde, et avec eux d'autres encore.

La seconde vague a vu le jour à la fin du XIX ${ }^{e}$ siècle après que l'Occident nous eut envahi, eut occupé notre pays, exporté sa culture et ses institutions et contrôlé nos politiques. Nous avons alors entrepris de défendre l'islam dans une tentative de renouveau de la pensée islamique afin de l'accorder aux conditions présentes (celles qui prévalaient à la fin du $\mathrm{XIX}^{\mathrm{e}}$ et au début du $\mathrm{XX}^{\mathrm{e}}$ siècle) tout en protégeant ses fondements... Al-Azhar s'est engagé dans ce mouvement de renouveau, qu'elle a dirigé en repoussant toutes les tentatives visant à ébranler les fondements de la religion islamique en tant que réfèrent régulateur des comportements humains et des relations entre individus ${ }^{\circ}$; cette tentative s'accordait avec la situation de l'islam à cette époque... à savoir, l'époque de la colonisation et de la résistance à la colonisation.

Après cela, lorsque la conception laïque a commencé à prévaloir dans nos institutions et nos relations juridiques, et, ce faisant, a commencé à régir aussi nos comportements individuels, sont apparues de nouvelles tentatives de renouveau de la pensée islamique accompagnées par des tentatives de retour du réfèrent islamique, afin qu'à nouveau il domine les systèmes politiques et sociaux et qu'il soit à nouveau reconnu comme source de la législation, des comportements et des relations humaines.

Au cours de chacune de ces étapes, la pensée islamique s'est trouvée confrontée à des problèmes qui se posaient à la communauté musulmane et auxquels elle a apporté des solutions appropriées ${ }^{\circ}$; il est à remarquer, toutefois, que certains domaines n'ont pas fait l'objet d'études suffisamment approfondies ni d'efforts suffisants, comme par exemple celui des relations internationales. Ainsi on peut se demander quelle serait notre classification des différents États. Devons-nous les classer en fonction de leur proximité ou de leur éloignement par rapport aux principes islamiques, ou en fonction de leur proximité ou de leur éloignement par rapport aux intérêts de la communauté musulmane $e^{\circ}$ ? Vous trouverez des nations qui sont éloignées du message de Dieu, mais plus proches de vous par les intérêts politiques et économiques, de même que vous trouverez d'autres pays qui, comme nous, ont connu l'expérience de la colonisation et qui font partie des pays reconnaissant la religion de Dieu. Je ne 
crois pas que beaucoup d'efforts aient été fournis en vue d'approfondir cette question.

S'agissant du domaine économique, il me semble que les efforts de la pensée islamique se sont essentiellement déployés dans deux domaines qu'ils ont traités de façon exhaustive ${ }^{\circ}$ : le premier concerne l'interdiction de l'usure (ribâ), le second l'obligation de l'aumône légale (zakât). Par contre, s'agissant des moyens permettant de développer l'économie conformément à la conception islamique, problème qui se situe entre ces deux questions, les efforts fournis pour approfondir ce domaine apparaissent comme étant nettement insuffisants.

Dans le même sens, s'agissant de la relation entre les textes et la réalité sociale... il apparaît que les textes clairs doivent obligatoirement être appliqués en tout temps et en tout lieu. Afin de faciliter leur mise en œuvre, nous devons faire en sorte que nos relations sociales et nos conditions d'organisation soient en mesure d'accueillir ces textes et qu'elles ne soient pas trop éloignées des possibilités de leur application pratique... Cette question exige de la part des sociologues de nombreuses études. Ainsi, par exemple, s'agissant de l'institution de l'héritage et des dispositions du figh relatifs à la famille, ils commandent une conception de la famille étendue qui est dès lors l'entité que nous devons préserver. Or, sur le plan social, faisons-nous des efforts suffisants pour préserver ces liens familiaux de façon à permettre une application adéquate et correcte de ces textes ${ }^{\circ}$ ?

Cette désagrégation que nous constatons aujourd'hui au niveau de la famille aura par la suite des conséquences allant dans le sens d'une remise en cause des principes se rapportant à cette question. Certains observent déjà que le fait pour les parents éloignés d'hériter n'est pas raisonnable dans la mesure où, pour eux, la famille type est la famille nucléaire, laquelle se compose du mari, de la femme et des enfants. $\mathrm{Ne}$ nous faut-il pas, au contraire, réfléchir aux moyens de soutenir, voire même de renforcer la famille élargie ${ }^{\circ}$ ? De telles questions exigent réflexion... Il est vrai que durant les cinquante dernières années, nous avons pu constater de réels efforts fournis par de nombreux penseurs dans ces domaines, toutefois nous ne saurions en rester là, et des efforts supplémentaires sont encore nécessaires. Nous nous sommes également engagés dans des controverses intellectuelles où nous avons traité de façon très générale ces questions au lieu d'entrer dans le détail... Je crois qu'il nous faut aujourd'hui nous arrêter afin de comprendre ce à quoi sont parvenus les efforts conduits au titre du renouveau dans les différents domaines des sciences sociales telles qu'abordées du point de vue de l'islam. Nous avons beaucoup de thèses de doctorat, d'études et autres ouvrages sur le sujet, qu'il nous faut maintenant rassembler afin d'en estimer le volume et l'apport exacts.

Al-Sha'b : Pensez-vous qu'il doive exister une institution qui s'occupe de ces interprétations et qui ait un droit de regard sur elles ${ }^{\circ}$ ?

T. al-Bishrî: Une institution ne s'occupant que d'ijtihâd est aujourd'hui devenue une nécessité, mais il ne s'agit pas de créer un organisme qui soit l'auteur de décisions religieuses ou qui vienne à monopoliser l'opinion en ce qui concerne les questions touchant à l'islam ou aux musulmans. L'une des raisons les plus importantes, qui explique que la pensée islamique soit toujours parvenue à se renouveler et qui plus est de façon spontanée, réside dans le fait que les initiatives de la umma ont été laissées sans monopole. En ce sens, l'instauration d'une institution est certes très importante et très utile quant à la spécialisation, la répartition du travail et 
l'harmonisation des efforts entre les différentes études, mais l'on ne saurait en aucun cas aboutir à une confiscation non plus qu'à une monopolisation, voire à l'établissement d'une autorité qui viendrait à accaparer l'opinion à l'exclusion de toute autre. Je pense que l'on ne peut échapper à la création d'une institution qui concentre les efforts fournis en la matière et qui favorise une sorte de spécialisation et de répartition du travail d'interprétation concernant tous ces domaines, mais il ne faut pas qu'il y ait à terme constitution d'un monopole de l'opinion islamique.

Al-Sha'b: La shari'a ${ }^{\circ}$ : Certains laïcs s'opposent à l'application de la sharîa invoquant son inadéquation à l'époque contemporaine...

T. al-Bishrî: Nous sommes tenus par le Coran et la Sunna et il s'agit là d'une obligation liée à la foi que l'on ne saurait enfreindre. Pour autant, il peut arriver que des dispositions contiennent un certain degré de divergence, comme cela s'est produit entre les différentes écoles. De fait, me semble-t-il, chacun des textes comportant des prescriptions - qu'il s'agisse du Coran ou de la Sunna - comportent des apparences de divergences en leur sein, il en va d'ailleurs de même en ce qui concerne le texte positif consacré par une loi ${ }^{\circ}$; de même, il peut arriver qu'au moment de leur application il y ait divergence entre le texte et des faits précis. C'est toujours en fonction du degré de divergence dans l'interprétation que contient le texte qu'il nous est possible de choisir entre les différentes opinions ce qui convient à nos situations sans enfreindre le texte ${ }^{\circ}$; ainsi, par exemple, si l'héritage de la femme était d'un huitième, cela signifie qu'il ne saurait être d'un sixième car il s'agit là d'une prescription déterminée..., la prohibition d'une chose ne saurait jamais devenir une permission, en revanche il peut arriver qu'il y ait divergence découlant du texte en rapport avec des faits précis, en ce cas là nous sommes dans le domaine de l'interprétation.

Al-Sha'b: La justice ${ }^{\circ}$ : Pensez-vous que le système juridictionnel actuel diffère du système juridictionnel défini dans l'islam?

T. al-Bishrî: Je ne pense pas que le système actuel diffère de beaucoup des fondements du système juridictionnel tel que conçu dans l'islam... ; je veux dire par là qu'il tend grandement vers la spécialisation et qu'il existe un principe connu du figh qui affirme la possibilité de spécialiser la justice par rapport au temps, en d'autres termes qui autorise le wâlî (gouverneur) à choisir le juge afin que celui-ci rende la justice pendant une période de temps déterminée, en principe quelques années, sans qu'il puisse y avoir prolongement au-delà de cette période ${ }^{\circ}$; dans le même sens, il peut y avoir spécialisation par rapport à un lieu, ce qui renvoie à ce que nous appelons aujourd'hui compétence territoriale, c'est-à-dire que le juge est compétent pour toutes les affaires survenues dans un ressort territorial déterminé; il existe enfin une spécialisation fondée sur l'affaire elle-même, que nous nommons la compétence matérielle ${ }^{\circ}$ : ainsi les affaires commerciales qui sont distinguées des affaires civiles, elles-mêmes séparées des affaires administratives qui sont elles aussi différenciées des affaires pénales. Il existe également une spécialisation fondée sur l'opinion, aux termes de laquelle il est fait obligation au juge de statuer conformément à l'opinion juridique d'une école particulière parmi les différentes écoles de figh, les justiciables devant toujours savoir quelle doctrine de quelle école leur est appliquée.

La spécialisation de la justice repose sur un fondement du figh et est donc parfaitement légale ${ }^{\circ}$; elle nous permet de tirer nos lois de la sharî́a islamique pour en 
faire des lois promulguées par l'État et appliquées par le juge, comme c'est aujourd'hui le cas en matière de droit de l'héritage, du testament, des biens waqf (biens de mainmorte) ou du droit du statut personnel.

De même encore, l'islam n'interdit pas qu'il y ait plus d'un juge chargé d'une affaire, tout comme il ne s'oppose pas à l'institution d'une justice à deux ou trois degrés plutôt que d'une justice à un seul degréo; tout cela dans le cadre d'une organisation juridictionnelle conforme à la shari'a. Pour ma part, il me semble qu'un tel système est plus précis et offre de meilleures garanties, surtout avec l'augmentation du nombre des affaires judiciaires et la multiplication des problèmes et litiges... La pluralité des juges et des degrés de juridictions me semble constituer un meilleur système doté d'une plus grande efficacité.

\section{Deuxième partie ${ }^{0}$ :}

\section{"Certains laïcs tentent d'exploiter le malaise des coptes et d'affronter par leur intermédiaire les mouvements islamiques, au lieu de se mesurer directement à eux sur le terrain intellectuel » (28 mars 1997)}

4 Le conseiller Târiq al-Bishrî se distingue d'autres penseurs par sa façon d'examiner les questions ayant trait à l'islam, notamment en recourant à une méthode de raisonnement qui consiste à considérer le sujet dans sa globalité, sous tous ses aspects, puis à émettre son opinion à partir d'une argumentation intellectuelle fondée sur des éléments solides puisés au réfèrent islamique. Vous vous trouvez ainsi en présence d'un homme qui vous éblouit par sa grande politesse et son extrême modestie, avec, en outre, l'impression d'être assis devant un « livre ouvert ", tant sa culture est vaste et puissante sa capacité à déduire des solutions à partir des dispositions de la shari'a pour ensuite les rapporter aux situations concrètes que nous vivons au quotidien. Il donne le sentiment, ce faisant, d'être une source à partir de laquelle semble toujours renaître la pensée islamique, et sans qu'il ait jamais épuisé toutes ses capacités.

Dans la première partie de cet entretien, publiée vendredi dernier [le 21 mars 1997], Târiq al-Bishrî a parlé de la nécessité de créer une instance chargée de veiller à l'interprétation portant sur toutes les questions liées à l'islam, sans toutefois instituer un monopole d'opinion. Il a également affirmé que l'hypothèse d'une contradiction entre les textes religieux et les intérêts de la société n'était pas envisageable, dans la mesure où la shari'a dans sa totalité protégeait ces intérêts. Il a démontré que l'islam incitait à l'interprétation, mais à partir des principes et sur labase des postulats contenus dans le Coran et la Sunna, ainsi que sur ceux issus des différentes exégèses.

6 Dans les propos d'aujourd'hui, nous allons suivre le conseiller al-Bishrî dans sa réflexion portant surtout ce qui touche aux droits des coptes appréhendés sous l'angle de la shari'a, de même que sur des questions ayant suscité la controverse, telles que le rapport entre shûrâ et démocratie, ou encore notre relation à l'Occident, voire enfin les raisons du recul enregistré dans le rôle assumé par al-Azhar. 
INDEX

Mots-clés : sharî̀a, droit islamique, droit 